\title{
Uncertainty measurement with the kinematic telescopic bar during industrial robot inaccuracy tests
}

\author{
Jerzy Józwik ${ }^{1, *}$, Elżbieta Jacniacka ${ }^{1}$ and, Dawid Ostrowski ${ }^{2}$ \\ ${ }^{1}$ Lublin University of Technology, Mechanical Engineering Faculty, Department of Production Engineering, 36 Nadbystrzycka Street, \\ 20-618 Lublin, Poland \\ ${ }^{2}$ The State School of Higher Education, The Institute of Technical Sciences and Aviation, 54 Pocztowa Street, 22-100 Chełm, Poland
}

\begin{abstract}
The subject of this article is the assessment of measurement uncertainty with the kinematic telescopic bar QC20-W in the accuracy test of MOTOMAN HP20 industrial robot. The measurements were performed to determine the statistical uncertainty of error measurement using the system applied. Analysed in tests was the robot's ability to recreate a circular outline through standard, complex and extended measurement of uncertainty measurement. The obtained results were served to perform rapid evaluation of robot inaccuracy. These uncertainties were based on the information included in the device calibration certificate (estimated with method B) but also on the basis of measurements and statistical data (estimated with method A). These components of the uncertainty budget take relatively small values $\left(\mathrm{u}_{\mathrm{c}}=0.818 \div\right.$ $4.130 ; \mathrm{U}=1.636 \div 8.260$ for $\mathrm{k}=2$, which proves that a properly selected method was applied to the research task. The method of research and calculation precisely identified key uncertainties allowing for an objective assessment of the industrial robot errors carried out with Renishaw the kinematic telescopic bar.
\end{abstract}

\section{Introduction}

Robot is an automatic device capable of handling operations in an industrial manufacturing process, equipped with a kinematic system consisting of at least three motion units and an autonomous programmable control system [1]. The cycle of manipulative and locomotive movements performed by the robot is most often reproducible, however, it may vary depending on the modification of the program, the information given or the state of the environment [1-3]. In industrial practice, robots perform movements with certain accuracy, which is variable during operation generating errors interfering of accuracy [4]. In the work [5] geometric and non-geometric errors are distinguished. Work [6] defines errors affecting the accuracy of a robot, dividing it into three basic groups: dynamic, structural, and kinematic. Another example of error classification is presented in [7], where four groups of industrial robot accuracy errors are distinguished: geometric, dynamic, thermal and system. There are few measuring systems that enable effective identification and evaluation of robot errors. The QC20-W Ballbar diagnostic system, dedicated to $\mathrm{CNC}$ machines, is used in this study. Although the use of the QC20-W Ballbar system for CNC machine tools is very wide $[1,4,8-10]$, in robots it is applied on a much lower scale [2, 11-14]. Some authors [11,12] compare the accuracy of selected industrial robots with the use of a ball-shaped, telescopic kinematic bar. ABB IRB 1600 industrial robots were used in various tests (YZ and $\mathrm{XZ}$ ) for variable radius values $(100,150$ and $300 \mathrm{~mm})$ and feed rates ranging from 20 to $700 \mathrm{~mm} / \mathrm{s}$. The measurement was taken at the selected point with defined coordinates $(0,65,149 \mathrm{~mm})$ in the robot base coordinate system. Based on the results, the authors $[11,12]$ determined the relationship between the programmed flow rate of the effector effect and the recorded deviations but did not determine measurement uncertainty. It is therefore possible to conclude that these results are not precise and are only subjective. Uncertainty of measurement, besides accuracy, repetition, resolution, or positioning errors, is one of the most important concepts in research procedures and in defining individual errors of industrial robots. [15] Measurement uncertainty is one of the most important tasks of utmost relevance to accurate measurement of a given quantity and includes a number of uncertainties regarding the impact of major factors influencing the measurement result [2, 15-19]. From literary analysis, it is clear that uncertainty is an interval that characterizes the scattering of values of a given quantity that may be reasonably attributed to the true value (measurand) calculated from the measured information. Uncertainty is defined as a certain "doubt" about the value of the measurement result. It is presented as the range around the measurement result, within which, with a certain probability, there lies the real value of the measured quantity (Fig. 1) [1, 2, 15-20].

\footnotetext{
${ }^{*}$ Corresponding author: j.jozwik@pollub.pl
} 


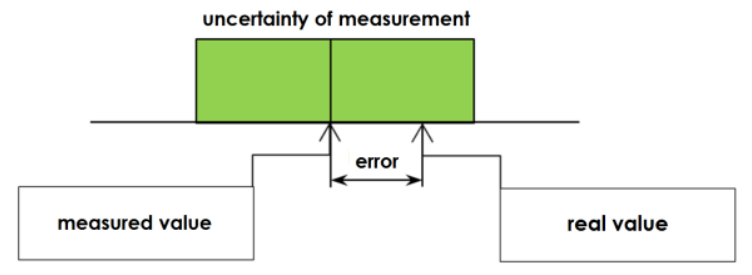

Fig. 1. Graphical representation of error and of measurement uncertainty [2].

Regarding the types of uncertainty, we distinguish standard uncertainty $\mathrm{u}$ or $\mathrm{u}(\mathrm{x})$, complex standard uncertainty $\mathrm{Uc}$, and expanded uncertainty $\mathrm{U}$ or $\mathrm{U}(\mathrm{x})$ or Uc (x). [15] The estimation of measurement uncertainty of an industrial robot presents a number of different difficulties, discussed in the literature $[2,8]$, the evaluation of which requires an experimental study [2, $15,19-26]$. Only proper estimation of uncertainty will allow for objective estimation of robot inaccuracies and selection of the optimum measurement method [1, 2]. The theory of measurement uncertainty has now replaced the theory of measurement accuracy (measurement error). Uncertainty theory is commonly recognized, universal and accepted by all metrology organizations, and therefore it is important to remember that accuracy of any measurement is described by its uncertainty [20-26].

\section{Research methodology}

\subsection{Research stands}

During experimental works required for the estimation of statistical components of the kinematic telescopic bar, the following positions and computerized measurement and calculation systems were used:

- industrial robot MOTOMAN HP20, as research object,

- measurement system QC20 Ballbar,

- calculation environment Excel,

- calculation environment Statistica,

- specialized software for data recording and analysis Ballbar 20 v.5.

\subsubsection{Object of research}

The object of the conducted research was measurement uncertainty assessed with the kinematic telescopic bar of circular displacement of a universal of a six-axis industrial robot MOTOMAN HP20 by Yaskawa (Fig. 2a). All MOTOMAN HP20 work axes are rotary axes. The ranges of movement of the particular axes of the robot are: $\mathrm{S}$ axis $\left( \pm 180^{\circ}\right)$, $\mathrm{L}$ axis $\left(+155 \%-110^{\circ}\right)$, $\mathrm{U}$ axis $\left(+255^{\circ} /-165^{\circ}\right), \mathrm{R}$ axis $\left.\left( \pm 200^{\circ}\right)+230 \%-50^{\circ}\right)$ and $\mathrm{T}$ axis $\left( \pm 360^{\circ}\right)$. The axis of rotation of joint 1 is vertical, while the axes of joints 2 and 3 are horizontal. The maximum distance in the axis perpendicular to the base plane is $3063 \mathrm{~mm}$, and in the axis parallel to the base plane $1717 \mathrm{~mm}$. Repeatability of the positioning based on the robot calibration certificate and the manufacturer's assessment is equal to $0.06 \mathrm{~mm}$. a)

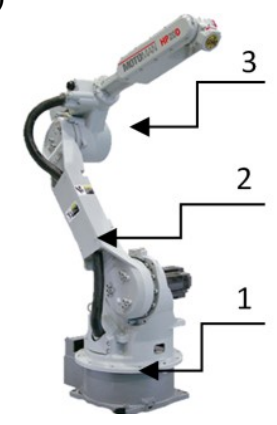

b)

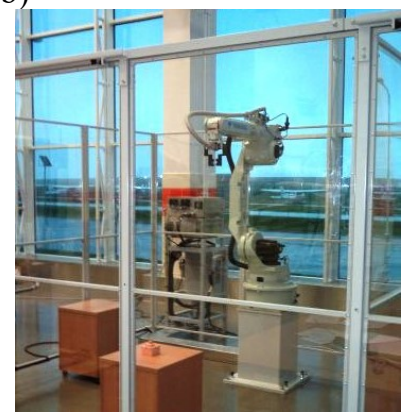

Fig. 2. Industrial robot HP20, a) general view, b) robot's positioning on research station, 1,2,3-robot connectors.

The maximum lifting capacity of the robot is $20 \mathrm{~kg}$. The robot is controlled by YASNAC FS 100 controller.

\subsubsection{Measurement set-up}

As part of the accuracy test of the Motoman HP20 industrial robot, Renishaw QC20-W Ballbar Telescopic Tester was used to identify non-measurable laser interferometer errors. The QC20-W Ballbar enables performing a circle test for different radius values in three planes. The detailed parameters of the measuring device are: resolution of the transducer $(0.1 \mu \mathrm{m})$, measurement accuracy $\pm(0.7+0.003 \mu$ L) $\mu \mathrm{m}$ in the temperature range of $15-25^{\circ} \mathrm{C}$, where $\mathrm{L}$ is the length at which the error is measured), maximal sampling rate $(1000 \mathrm{~Hz})$, data transmission range $(10 \mathrm{~m}$, Bluetooth Class 2), operating temperature $\left(0-40^{\circ} \mathrm{C}\right)$. The basic elements of the system are:

- a $100 \mathrm{~mm}$ long kinematic ball bar assembly,

- a set of extension rods with lengths of 50, 150 and $300 \mathrm{~mm}$,

- magnetic handles,

- a calibrator,

- software for recording and analysis of data.

The measurement consists in registering the deviation of the circle while performing the path by an effector on a circular path in the selected plane for a fixed location and speed of movement. The magnetic grip of the effector (1) is attached to the robot gripper and the magnetic base (4) is placed on the table prepared for this purpose. Between the elements (1) and (4), a displacement transducer (3) of a specified length is placed to measure the deviations of radius displacement over the whole range of circular paths.

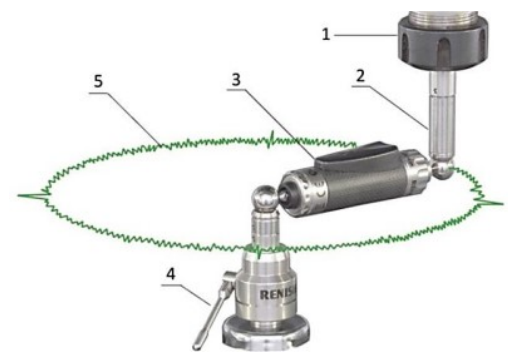

Fig. 3. Test using a measuring system QC 20 Ballbar, 1 - effector's impact sleeve, 2 - magnetic gripper, 3 - displacement transducer, 4 - magnetic base, 5 - circular measuring path. 
Although the method is dedicated for $\mathrm{CNC}$ machine tools, to some extent and with some restrictions it can also apply to measuring accuracy of industrial robots, as demonstrated by the authors of these works [2, 5, 11 , $12]$.

\subsection{Test settings}

The QC20-W Ballbar kinematic bar allows for identification of errors typical for numerically controlled machine tools such as: roundness deviation, slacks (reverse, transverse), lateral and parallel deviations, and many others; however, some of them may as well describe the properties of industrial robot. The authors point to the deviation of the circle as a representative measure of robot inaccuracies in a dynamic test during motion describing the ability of a path to follow on a circular outline. It should be pointed out that this type of path is often used when working in an industrial environment. Measurement using the QC20 Ballbar can be carried out in one of the three planes XY, XZ and YZ. As mentioned in section. 2.1.1, the first step prior to accession to the main works, was the establishment of research methodology. The measurement consisted in recording the radius deviation for a given radius while performing a circular path by an effector in a given plane for the selected path and feed value (Fig. 4). In order to ensure a constant linear velocity of the effector flow $v_{f}$, according to the recommended procedure, each programmed measurement motion was preceded by a runoff and a freefall. Measurements were carried out in the $\mathrm{XY}$ plane in the $0-360^{\circ}$ range while moving clockwise CW and counter clockwise CCW (Fig. 4).
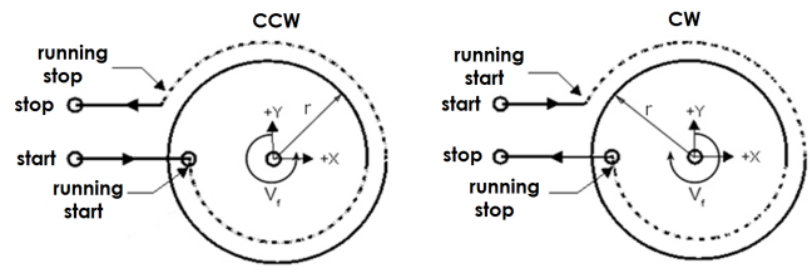

Fig 4. Method for accuracy measurement of HP20 robot with a telescopic kinematic bar QC20-W Ballbar by Renishaw [2].

The circle test was performed for three radius values $r$ of the interpolated circle, equal to: $100 \mathrm{~mm}, 150 \mathrm{~mm}$ and $300 \mathrm{~mm}$. The speed effector movement $v_{f}$ was set at the levels of: $50 \mathrm{~mm} / \mathrm{s}, 75 \mathrm{~mm} / \mathrm{s}, 100 \mathrm{~mm} / \mathrm{s}$. For each test configuration, three repetitions were performed. Each change of length of the QC20 Ballbar telescopic bar was preceded by a calibration of the length of the bar arm. In the paper, the authors proposed their own research methodology, taking into account the measurement capabilities of the applied system and the specificity of the robot's operation. This methodology is optimized for the duration of the test and the possibility of estimating the inaccuracy of the robot in industrial conditions. Figure 5 shows the method for attaching a telescopic kinematic bar to a measuring stand. The magnetic centring base (2) is mounted in the robot axis on the metal element (1) placed on the table (in front of the robot) in the central part of the HP20 work area. This location of the centre of the interpolated circle in the base coordinate system of the robot is dictated by the most commonly used area of the robot. This does not mean, however, that this is the position in which the greatest value of the identified errors should be expected. The telescopic kinematic bar (3) was placed between the magnetic base (2) and the magnetic holder (4) attached to the robot's gripper (effector) (5). A computer with specialized Ballbar 20 V5 software was used to record measurement data, and was connected to a measuring device via Bluetooth.

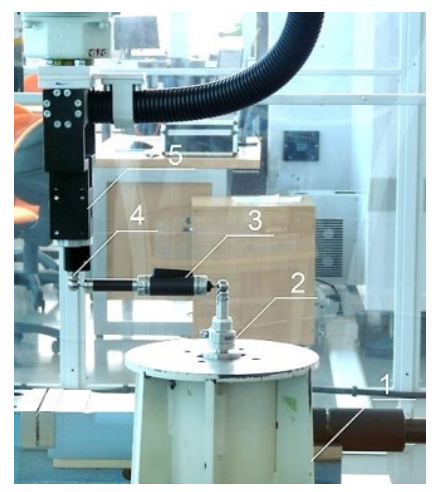

Fig. 5. Method of attachment of the QC20-W Ballbar to the test position, where: 1 - metal element, 2 - centring magnetic base, 3 - telescopic kinematic bar, 4 - magnetic holder, 5 - robot's gripper (effector).
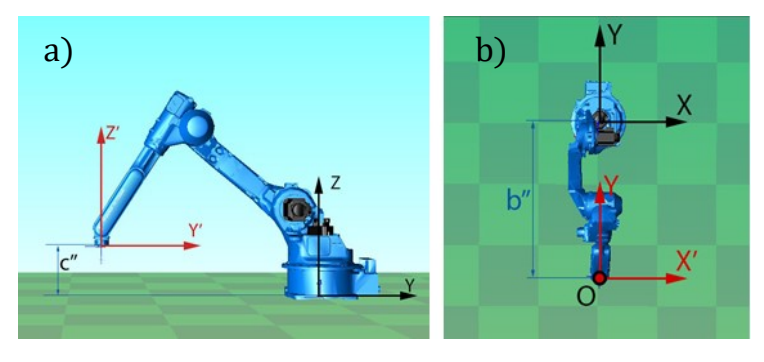

Fig. 6. Location of the centre of the circle in the base coordinate system of the robot: a) side view, b) top view.

In Fig. 6, the letter $\mathrm{O}$ denotes the centre point in the base coordinate system, with respect to which the position of the radius for realizing the circular outline path during the test is defined. The offset of the centre point in the Cartesian coordinate system relative to the beginning of the coordinate system of the studied robot were $b^{\prime \prime}=750 \mathrm{~mm}$ in the $\mathrm{Y}$ axis and $\mathrm{c}^{\prime \prime}=200 \mathrm{~mm}$ in the $\mathrm{Z}$ axis, respectively. Measurement conditions, i.e. temperature, humidity and pressure were monitored and maintained on a constant level. The trials were conducted according to ISO 230-4 standard. Ballbar 20 V5 software was used to record measurement data, generate graphs and in extensive statistical analysis of results based on the ISO standard applied.

\section{Test results}

Considering the construction of the robot, the evaluation of the results of research with the kinematic telescopic bar was aimed at the analysis of universal inaccuracy measurements, the most important of which is the 
deviation of the circle and the path of the circular profile. The analysis of errors identified by the kinematic telescopic bar showed that most of them refer to the axis of the Cartesian coordinate system of CNC machine tools. Due to the fact that the errors identified by the kinematic telescopic bar may arise for reasons that do not occur in relation to robots (e.g. non-uniform stress of straight edge (no straight edge), mismatched parameters of the linear error compensation, overheating or damage to screws in one of the axes (lack of such screws) or misalignment of the guides), the authors focused on general measures of inaccuracy of industrial robots. In particular, they demonstrated the possibility of dynamic measurement of roundness deviation, which is a big advantage of the system and allows assessing the circular trajectory of a given radius in any angular position. For each measurement configuration, i.e. the radius $r$ value, the path with a circular outline $r$ and the linear velocity of the robot's effector $v_{f}$, experimental deviation of the circle was determined. Figure 7 presents an exemplary map of deviation of the circle in full angle defined for the measurement circle $r=100 \mathrm{~mm}$ and the constant linear velocity of the effector motion $v_{f}=50 \mathrm{~mm} / \mathrm{s}$.

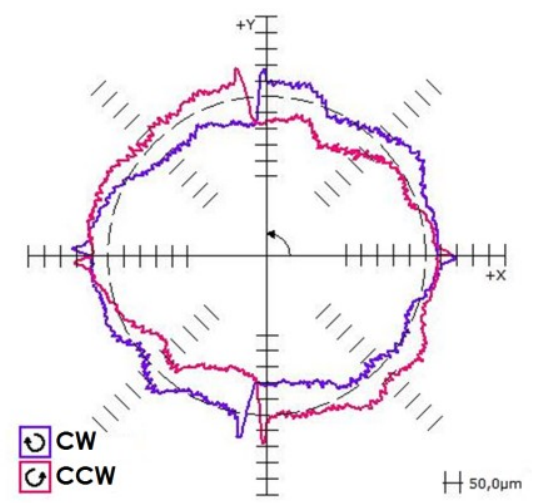

Fig. 7. Maps of the roundness deviation $\Delta \mathrm{o}$ for the interpolated circle with a radius $r=100 \mathrm{~mm}$ and $\mathrm{vf}=50 \mathrm{~mm} / \mathrm{s}$.

The maps of the roundness deviation test for all conducted tests indicate "jumps" characteristic of backlash. The shape of the actual interpolated circle observed in test may indicate that there are loops in the kinematic arms of the robot related to their insufficient rigidity. The graphs also show the areas in which the deviation of the radius of amplitude is increased, especially at higher feed rates. This fact may be a sign of vibration during effector movement. It should be borne in mind, however, that while measurements using the QC20 Ballbar telescopic bar are local and refer to numerically controlled machine tools, the kinematic structure of industrial articulated robots is more complex. Therefore, full evaluation of accuracy requires examination of the whole robot work space. Also, the way of interpreting the different types of errors as well as their types should be adequate for the construction of the robot, its weight and working conditions. As with laser interferometer measurements, QC20 Ballbar testing should be performed when the robot is in a steady state. For this reason, only deviation of roundness $\Delta_{0}$ was assessed in the work.
The average value of roundness deviation $\Delta_{o}$ in the function of linear velocity of tool movement $v_{f}$ and radius length $r$ of the interpolated circle was presented in Figure 8.

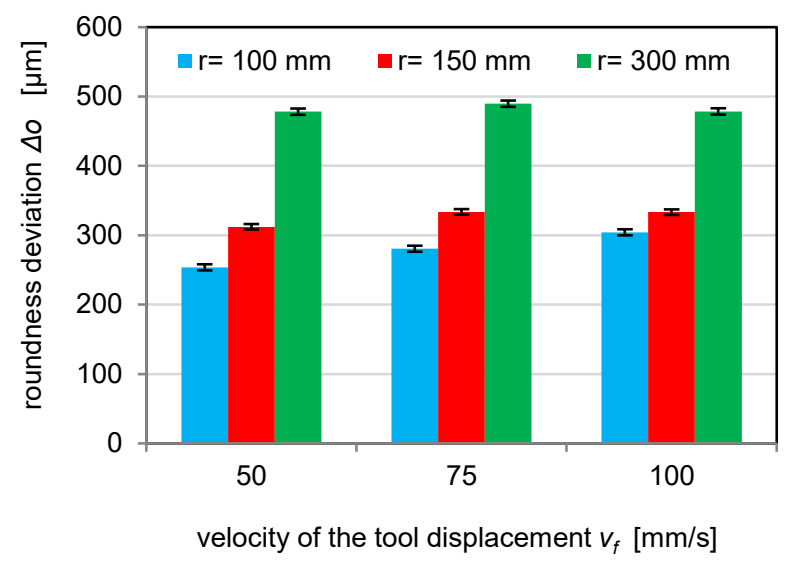

Fig. 8. Mean value of roundness deviation as a function of the linear velocity of the tool displacement $v_{f}$ and the length of the $r$ radius of the interpolated circle.

\section{Estimation of uncertainty}

The measurement uncertainty recorded with the QC20W Ballbar the kinematic telescopic bar includes a number of component uncertainties, which take into account the effect of all errors affecting the measured result. As for laser interferometers, also for QC20-W Ballbar kinematics, these uncertainties are estimated based on the information contained in the calibration certificate of the instrument, but also on the basis of measurements and statistics. Table 1 presents the errors and uncertainties used in system testing. These data are based on QC20-W Ballbar calibration certificate. The other components of the kinematic measurement uncertainty of the telescopic bar were estimated on the basis of the results obtained with method A and nonstatistical components were estimated with method B.

Table 1. The errors and inaccuracies of the kinematic telescopic bar used in the tests, determined on the basis of a calibration certificate (at an air temperature of $19.3{ }^{\circ} \mathrm{C}$, 1029 mbar, relative humidity $42.3 \% \mathrm{RH}$ ).

\begin{tabular}{|l|r|}
\hline Positioning error & $+/-0.5 \mu \mathrm{m}$ \\
\hline Maximum error & $+/-0.19 \mu \mathrm{m}$ \\
\hline Uncertainty $(\mathrm{k}=2)$ & $0.24 \mu \mathrm{m}$ \\
\hline $\begin{array}{l}\text { Uncertainty of pattern, for Zerodur } \\
\text { during calibration of length: } 50, \\
100,150,300(\mathrm{k}=2)\end{array}$ & $0.00085 \mu \mathrm{m}$ \\
\hline
\end{tabular}

Extended measurement uncertainty $U$ using QC20 Ballbar system was determined from the equation (1).

$$
U= \pm u_{c} k
$$

where: $u_{c}$ - complex standard uncertainty, $\mathrm{k}$ - expansion factor. 
Complex standard uncertainty $u_{c}$ was derived from equation (2), including component $u_{A}$ from the scattering of the measurement result estimated with method A (3), and the components of measurement uncertainty determined with method $\mathrm{B}$, obtained from the nonstatistic sources (4).

$$
u_{c}= \pm \sqrt{u_{A}^{2}+\sum_{i=1}^{n} u_{B i}^{2}}
$$

where: $u_{A}$ - standard uncertainty estimated with the method A (statistical), $u_{B i}$ - standard uncertainties nonstatistical, estimated with method B

$$
u_{A}= \pm \sqrt{\frac{\sum\left(x_{i}-\bar{x}\right)^{2}}{n(n-1)}}
$$

where: $x_{i}$ - single measurement result, $\bar{x}$ - average measurement results, $\mathrm{n}$ - number of measurements

Non-statistical components were designated as the sum of centring uncertainties $u_{B P}$, calibration uncertainties $u_{B K}$ and calibration error $u_{B E}$. Equation (4) describes the sum of squares of uncertainties estimated by B method.

$$
\sum_{i=1}^{n} u_{B i}^{2}=u_{B P}^{2}+u_{B K}^{2}+u_{B E}^{2}
$$

Taking into account the above, the complex standard measurement uncertainty with the kinematic telescopic bar was determined from the relationship (5):

$$
u_{c}= \pm \sqrt{u_{A}^{2}+u_{B P}^{2}+u_{B K}^{2}+u_{B E}^{2}}
$$

Each component was determined with consideration of the data included in the system calibration certificate. Therefore, when accepting a rectangular distribution of probability, component $u_{B P}$ was determined as (6), and component $u_{B K}$ was determined from equation (7), with the assumption of normal distribution. Component $u_{B E}$ was determined from equation (8) assuming a rectangular probability distribution.

$$
\begin{aligned}
& u_{B P}= \pm \frac{0.5}{\sqrt{3}}[\mu \mathrm{m}] \\
& u_{B K}= \pm \frac{0.24}{2}[\mu \mathrm{m}] \\
& u_{B E}= \pm \frac{0.19}{\sqrt{3}}[\mu \mathrm{m}]
\end{aligned}
$$

In the analysed case, the uncertainty resulting from the temperature measurement and the coefficient of thermal expansion were omitted. The assumption of the negligence of the omitted uncertainties was taken into account because of the direct calibration of the QC20 Ballbar the kinematic telescopic bar prior to the measurement itself and a very short measurement time of several minutes. The authors of the study, performing the measurement in thermally stable conditions, where the ambient temperature did not change by more than $0.2{ }^{\circ} \mathrm{C}$, assumed that during the measurement no significant changes in the length of the measuring arm during the test would occur. Thus, given the above, these uncertainties were considered insignificant. The uncertainty surrounding the pattern from the Zerodur was also omitted. The basis for not taking into account the uncertainty of extending the pattern is its extremely low (near zero) coefficient of thermal expansion. This coefficient is practically independent of the temperature,

\begin{tabular}{|c|c|c|c|}
\hline \multirow{3}{*}{ 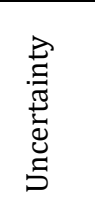 } & \multicolumn{3}{|c|}{$\mathrm{R}=100 \mathrm{~mm}$} \\
\hline & $\begin{array}{c}V_{f}=50 \\
\mathrm{~mm} / \mathrm{min}\end{array}$ & $\begin{array}{c}V_{f}=75 \\
\mathrm{~mm} / \mathrm{min}\end{array}$ & $\begin{array}{c}V_{f}=100 \\
\mathrm{~mm} / \mathrm{min}\end{array}$ \\
\hline & \multicolumn{3}{|c|}{ Range of uncertainty values $[\mu \mathrm{m}]$} \\
\hline$u_{A}$ & \pm 4.116 & \pm 3.648 & \pm 1.304 \\
\hline$u_{B P}$ & \pm 0.289 & \pm 0.289 & \pm 0.289 \\
\hline$u_{B K}$ & \pm 0.120 & \pm 0.120 & \pm 0.120 \\
\hline$u_{B E}$ & \pm 0.110 & \pm 0.110 & \pm 0.110 \\
\hline$u_{c}$ & \pm 4.130 & \pm 3.663 & \pm 1.345 \\
\hline \multirow[t]{2}{*}{$\begin{array}{l}U \\
(k=2)\end{array}$} & \pm 8.260 & \pm 7.326 & \pm 2.690 \\
\hline & \multicolumn{3}{|c|}{$\mathrm{R}=150 \mathrm{~mm}$} \\
\hline$u_{A}$ & \pm 1.689 & \pm 1.348 & \pm 0.748 \\
\hline$u_{B P}$ & \pm 0.289 & \pm 0.289 & \pm 0.289 \\
\hline$u_{B K}$ & \pm 0.120 & \pm 0.120 & \pm 0.120 \\
\hline$u_{B E}$ & \pm 0.110 & \pm 0.110 & \pm 0.110 \\
\hline$u_{c}$ & \pm 1.721 & \pm 1.388 & \pm 0.818 \\
\hline \multirow[t]{2}{*}{$\begin{array}{l}U \\
(k=2)\end{array}$} & \pm 3.442 & \pm 2.776 & \pm 1.636 \\
\hline & \multicolumn{3}{|c|}{$\mathrm{R}=300 \mathrm{~mm}$} \\
\hline$u_{A}$ & \pm 1.205 & \pm 2.317 & \pm 3.846 \\
\hline$u_{B P}$ & \pm 0.289 & \pm 0.289 & \pm 0.289 \\
\hline$u_{B K}$ & \pm 0.120 & \pm 0.120 & \pm 0.120 \\
\hline$u_{B E}$ & \pm 0.110 & \pm 0.110 & \pm 0.110 \\
\hline$u_{c}$ & \pm 1.250 & \pm 2.340 & \pm 3.860 \\
\hline $\begin{array}{l}U \\
(k=2)\end{array}$ & \pm 2.500 & \pm 4.680 & \pm 7.720 \\
\hline
\end{tabular}
especially in the study range (Zerodur in the range 0 to $50{ }^{\circ} \mathrm{C}$ has an average thermal expansion at the level 0 $\left.\pm 0.007 \times 10^{-6} \mathrm{~K}^{-1}\right)$.

Table 2. Uncertainty and its components when measuring deviation of the arm circumference of the test robot with the kinematic telescopic bar.

Based on the assumptions adopted in the paper, computational formulas were developed and the values of individual components of uncertainty determined. Uncertainty and its components at $\mathrm{k}=2$ for kinematic deviation of the telescopic bar for the test arm length $r=$ $100 \mathrm{~mm}, \mathrm{r}=150 \mathrm{~mm}$ and $\mathrm{r}=300 \mathrm{~mm}$, and the armature effect velocity equal to $v_{f}(50,75$ and $100 \mathrm{~mm} / \mathrm{min})$ were included in Table 2. Table 2 clearly indicates that the dominant component of the uncertainty budget for measurement with the kinematic telescopic bar is standard uncertainty $u_{A}$ (statistical component resulting from the scattering of measurement result) and the uncertainty of centring the QC20-W Ballbar on the station $u_{B P}$. The consequence of the high value of standard uncertainty is the value of complex standard uncertainty $u_{c}$ and extended uncertainty $U$. Other components of uncertainty, i.e. uncertainty of calibration $u_{B K}$ and calibration error $u_{B E}$ only slightly affect the value of the complex standard uncertainty $u_{c}$ and expanded uncertainty $U$. The results presented in Table 2 appear to indicate that for most of the studied cases $(r=$ $100 \mathrm{~mm}$ and $\mathrm{r}=250 \mathrm{~mm}) v_{f}$ of the robot during circular 
interpolation implies a decreasing tendency to change the uncertainty of measurement, which means that its accuracy increases. It should be added that any result of the deviation of the roundness in the measured operating conditions should be recorded with the appropriate (for the arm $r$ and effector movement velocity $v_{f}$ ) uncertainty.

\section{Conclusion}

The analysis of uncertainty of kinematic measurement taken with the QC 20-W Ballbar telescopic bar show that components of the uncertainty budget take relatively small values $\quad\left(u_{c}=0.818 \div 4.130\right.$; $U=1.636 \div 8.260$ for $k=2$, which proves that a properly selected method was applied to the research task. Focusing on universal inaccuracies (roundness deviation) and the benefits of the QC 20-W Ballbar system for industrial robots enables evaluation of uncertainty of a circular trajectory of a given radius. The ability to identify circular deviation for different radius values of an interpolated circle allows an assessment of the impact of measuring length in the industrial robot space (from one attachment) to the uncertainty value of the measurement in all perpendicular planes, without changing both the program and the bar orientation. This significantly shortens the test run and the ability to assess standard uncertainty.

It should be noted that QC 20-W Ballbar the kinematic telescopic bar measurements are performed as dynamic measurements, i.e. in certain positions and typically without load on the robotic arm of the displaced elements.

Based on the results of the research and calculation, it can be concluded that the kinematic telescopic bar test results are subject to very low measurement uncertainty and therefore high accuracy.

\section{References}

1. J. Honczarenko, Roboty przemysłowe. Budowa i zastosowanie (WNT, Warszawa, 2010)

2. J. Józwik, D. Ostrowski, Wybrane problemy badawcze robotów przemysłowych (Wydawca Politechnika Lubelska, 2016)

3. W. Zhenhua, X. Hui, Ch. Guodong, S. Rongchuan, L. Sun, Ind. Rob. 41, 5 (2014)

4. J. Józwik, P. Pieśko, G. Krajewski, Maintenance and Reliability, 47, 3 (2010)

5. M. Abderrahim, A. Khamis, S. Garrido, L. Moreno,Accuracy and Calibration Issues of
Industrial Manipulators (Industrial Robotics Programming, Simulation and Applications, 2006)

6. K.L. Conrad, P.S. Shiakolas, T.C. Yih, Robotic calibration issues: accuracy repeatability and calibration (Proceedings of the 8th Mediterranean Conference on Control \& Automation, Rio, Patras, Greece, 2000)

7. B. Greenway, Ind. Rob. 27, 4 (2000)

8. J. Józwik, I. Kuric, L. Semotiuk, Communications 3A (2014)

9. J. Józwik, M. Czwarnowski, Adv. Sci. Technol. Res. J. 9, 28 (2015)

10. J. Józwik, L. Semotiuk, I. Kuric, Adv. Sci. Technol. Res. J. 9, 28 (2015)

11. M. Slamani, A. Joubair, I. Bonev, Ind. Rob. 42, 6 (2015)

12. M. Slamani, A. Nubiola, I. Bonev, Ind. Rob. 39, 1 (2012)

13. K. Young, C.G. Pickin, Ind. Rob. 39, 6 (2000)

14. B. Shirinzadeh, Ind. Rob. 25, 1 (1998)

15. P. Majda, D. Rozkrut, Adv. Manuf. Sci. Technol. 39, 3 (2015)

16. G.M. Krolczyk, J.B. Krolczyk, R.W. Maruda, S. Legutko, M. Tomaszewski, Measurement $\mathbf{8 8}$ (2016)

17. A. Glowacz, Z. Glowacz, Infrared. Phys. Technol. 81 (2017)

18. E. Feldshtein, J. Józwik, S. Legutko, Adv. Sci. Technol. Res. J. 10, 30 (2016)

19. G. Alici, B. Shirinzadeh, Mech. Mach. Theory 40, 8 (2005)

20. A. Angelidis, G.Ch.Vosniakos, Int. J. Precis. Eng. Man. 15, 1 (2014)

21. C. Gong, J. Yuan, J.Ni, Int. J. Mach. Tool Manu. 40, 14 (2012)

22. P.S. Shiakolas, K.L. Conrad, T.C. Yih, Int. J. Model. Simul. 22, 3 (2002)

23. J. Swevers, W. Verdonck, J.DeSchutter, IEEE Contr. Syst. Mag. 27, 5 (2007)

24. G.M. Krolczyk, P. Nieslony, J.B. Krolczyk, I. Samardzic, S. Legutko, S. Hloch, S. Barrans, R.W. Maruda, Measurement 70 (2015)

25. P. Nieslony, G.M. Krolczyk, K. Zak, R.W. Maruda, S. Legutko, Precis. Eng. 47 (2017)

26. G.M. Krolczyk., R.W. Maruda, P. Nieslony, M. Wieczorowski, Measurement 94 (2016) 